\title{
Short term effect of integrated Yoga on anxiety, stress and heart rate variability in first year medical student
}

\author{
Sumitra Sudharkodhy ${ }^{1, *}$, Karthiyanee Kutty ${ }^{2}$, N J Patil ${ }^{3}$, Vinutha Shankar ${ }^{4}$ \\ ${ }^{\mathbf{1}}$ Assistant Professor, Dept. of Physiology, Karpaga Vinayagar Institute of Medical Sciences, Tamil Nadu, \\ ${ }^{2,4}$ Professor, Dept. of Physiology, ${ }^{3}$ Associate Professor \& HOD, Dept. of Integrative Medicine, Sri Devaraj Urs \\ Academy of higher education and Research, Kolar, Karnataka, India
}

*Corresponding Author:

Email: sumitradoctor@gmail.com

\begin{abstract}
Introduction: Medical students are exposed to various stresses, which is known to affect cardiovascular system which can be assessed by noting the heart rate variability (HRV).

Aims: To assess the anxiety, stress and HRV in first year medical students and study the effect of one month integrated yoga intervention on them.

Materials and Methods: Spielberger state-trait anxiety inventory (STAI) and Perceived stress scale (PSS) were administered to 150 first year medical students. 60 students with a high anxiety and stress score were selected on the basis of specific inclusion and exclusion criteria and divided into study and control groups. A baseline HRV was documented on all 60 students. 30 students in the study group underwent supervised yoga intervention for one month. Post interventional assessments of anxiety, stress and HRV were done in both groups (study and control group). Paired t test was used to determine the statistical difference in pre and post interventions and independent $t$ test was used to determine the statistical difference between control and study groups.

Results: There was significant decrease in anxiety state and perceived stress with yoga intervention. Heart rate variability revealed that SDNN, RMSSD, Mean HR were significantly higher and LF, LF/HF ratio were significantly reduced after one month practice of yoga showing a shift towards parasympathetic dominance.

Conclusions: Integrated yoga intervention showed significantly decreases in state anxiety and perceived stress, and a shift towards parasympathetic dominance in heart rate variability. However, the anxiety trait didn't show significant improvement.
\end{abstract}

Received: $4^{\text {th }}$ November, 2017

Accepted: $15^{\text {th }}$ November, 2017

\section{Introduction}

Stress is a stimulus, either internal or external, that activates the hypothalamic pituitary adrenal (HPA) axis and the sympathetic nervous system resulting in physiological change. Longterm exposure to stressors can lead to various changes like depression, post-traumatic stress disorder, anxiety disorders and cardiovascular morbidity. ${ }^{1}$

In medical college, especially first year medical students are exposed to various stresses like demanding medical education, different teaching protocol and academic examinations which results into high levels of distress like depression and even suicidal thoughts. ${ }^{2,3}$ However academic examinations increases the levels of anxiety and emotional distress, which promotes diverse endocrine alterations including catecholamine, thyroid, and glucocorticoid effects. $^{4}$

Anxiety leads to autonomic imbalance resulting in decrease in heart rate variability (HRV) which is a potential risk factor for hypertension and atherogenesis which progress to coronary artery disease. ${ }^{5,6} \mathrm{HRV}$ is the temporal variation in consecutive heart beats measured from a standard electrocardiogram (ECG) and the assessment of this difference is performed in time domain and frequency domain. Decreased HRV means that there is a sympatho-vagal imbalance with a tilt towards sympathetic predominance or a reduction in parasympathetic activity.

Stress management is required for medical students to decrease depression and anxiety. Yoga is an ancient science established in India which gives the practitioner not only a sound body but also a sound mindand has shown to have effects on most physiological systems of the body. ${ }^{7,8}$ It is known to modulate autonomic output and helps achieve and maintain autonomic balance between two components (sympathetic and parasympathetic) of autonomic nervous system and stress. ${ }^{9}$

This study is designed to demonstrate the presence of stress, anxiety in first year medical students and associated with altered heart variability, and the role of yoga as an effective intervention to reduce stress, anxiety and improve HRV. 


\section{Materials and Methods}

Source of data: The subjects were first M.B.B.S student's volunteers staying in hostel/campus from Sri DevarajUrs medical college, Tamaka, Kolar, Karnataka. Sample size consists of 60 first year students (29 males and 31 females) in the age group of 18 to 20 years were recruited for the study. The protocol was approved by ethical committee of the institute (reference no: DMC/KLR/MEU/IEC-CER/30/2013-14).

Study protocol: It's a cross sectional prospective study. After written informed consent, 150 first year medical students were administered pretested and prevalidated Spielberger state-trait anxiety inventory (STAI) and perceived stress scale questionnaires. The questionnaires were analysed, and 60 students with Spielberger state-trait anxiety inventory (STAI) score above 40 and Perceived stress scale (PSS) score above 12 were included in the study. Students practicing any known stress relieving or relaxation techniques, on any drugs which affect the autonomic nervous system like anticholinergic, sympathomimetic, sympatholytic, having any major illness which is known to affect the autonomic nervous system were excluded. Complete clinical examination, including general and systemic examination was done. Anthropometric measurements recorded were height to the nearest centimeter using a stadiometer, and weight to the nearest kilogram (Table II).

30 students who volunteered to practice 4 weeks of yoga were assigned to study group and other 30 were the control group. Heart rate variability was recorded as baseline parameter. The study group were given a yoga intervention for 4 weeks, for 45 minutes a day, for 5 days a week under the supervision of a yoga instructor (Table I). The control group followed their normal daily routine and didn't practice any of the yogic techniques practiced by study group. At the end of 4 weeks, Spielberger state-trait anxiety inventory (STAI) and perceived stress scale questionnaires were administered and HRV was recorded and analysed using HRV analysis software.The details of protocol, data acquisitions and analysis are shown in Fig. 1.

Table I: Integrated yoga with duration of each

\begin{tabular}{|c|l|c|}
\hline S. No & Integrated yoga practices & Time (Minutes) \\
\hline 1 & Loosening exercises & 8 \\
\hline 2 & Suryanamaskara 12 steps & 15 \\
\hline 3 & Instant Relaxation Technique (IRT) & 2 \\
\hline 4 & Kapahalabhati- 3 rounds & 5 \\
\hline 5 & Nadisuddi pranayama & 5 \\
\hline 6 & Deep Relaxation Technique (DRT) & 10 \\
\hline 7 & Total & 45 \\
\hline
\end{tabular}

Table II: Baseline demographic profile of the subjects participated in the study ( $n=60$ )

\begin{tabular}{|l|c|c|c|}
\hline Parameters & $\begin{array}{l}\text { Control group } \\
(\mathbf{n = 3 0})\end{array}$ & $\begin{array}{l}\text { Study group } \\
(\mathbf{n = 3 0})\end{array}$ & P value \\
\hline Age ( years) & $18.05 \pm 0.21$ & $18 \pm 0.21$ & $>0.05$ \\
\hline Height $($ meters $)$ & $1.57 \pm 0.06$ & $1.55 \pm 0.06$ & $>0.05$ \\
\hline Weight $(\mathrm{kg})$ & $62.15 \pm 14.7$ & $58 \pm 11.3$ & $>0.05$ \\
\hline BMI $(\mathrm{kg} / \mathrm{m} 2)$ & $23.44 \pm 4.28$ & $22.91 \pm 4.36$ & $>0.05$ \\
\hline
\end{tabular}

All values are mean $\pm \mathrm{SD}, \mathrm{P}>0.05$ non-significant, $\mathrm{BMI}$ : Body mass index 


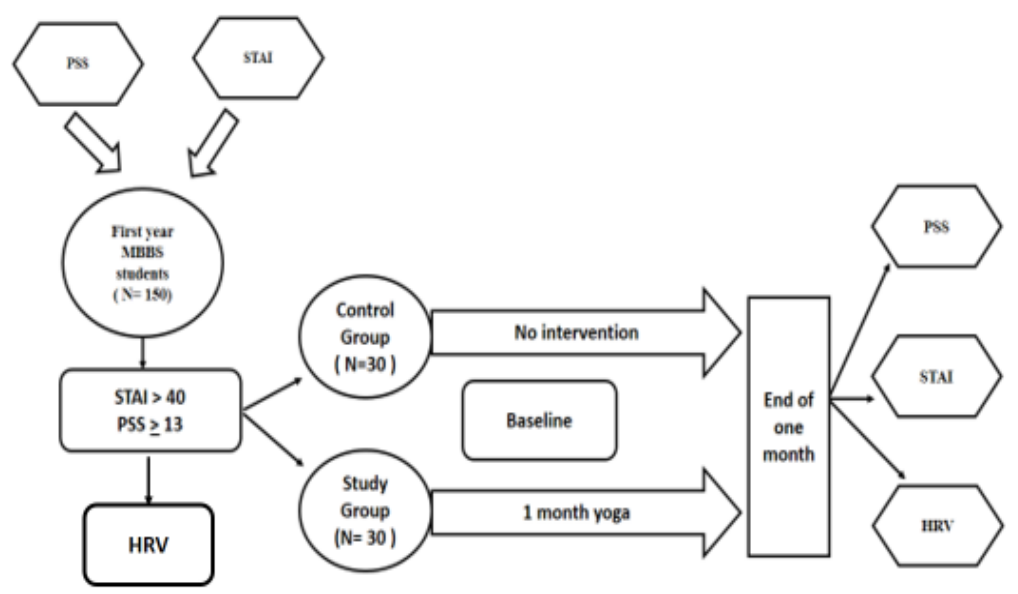

Fig. 1: Study Protocol for data acquisition and analysis

\section{Assessments}

State-trait anxiety inventory: The state trait anxiety inventoryfor adults (STAI-A) is the questionnaire which measures anxiety in adults. It measures both state anxiety and trait anxiety. The STAI has forty questions with four responses to each. The questionnaire were scored by adding the weighted (1 to 4 ) scores of each item, using the directions and scoring key provided in the manual for state-trait inventory (Form Y). The scores could vary from a maximum of 80 to a minimum of 80 . Internal consistency coefficients for the scale have ranged from 0.86 to 0.95 ; test-retest reliability coefficients have ranged from 0.65 to 0.75 over a 2-month interval (Spielberger et al., 1983).

Perceived stress score: The PSS-10 measures the psychological stress over the previous week with a 5 -point like rt scale $(0=$ never, $1=$ almost never, $2=$ sometimes, $3=$ fairly often, $4=$ very often). PSS-10 scores are obtained by reversing the response and then summing across all scale items. The scores range from $0-40$, with higher scores indicating greater stress. Scores around 13 are considered average. Scores of 20 or higher are considered high stress.Cronbach's alpha of the PSS-14 was >0.70.

Heart rate variability: It's beat to beat variation in heart rate under resting condition. These beat to beat variations occur due to continuous changes in the autonomic system outflow to the heart. Though HRV can be measured over any length of time of recorded ECG, as per task force guidelines (1996) at least five minutes of ECG must be recorded to quantify sympathetic and parasympathetic tone.

1. Procedure: Following instructions are given to the subjects:

a. subject should not have coffee, nicotine or alcohol 24 hours prior to testing; (ii) To avoid meals preceding 2 hours of the testing; (iii) Drugs which affect cardiovascular autonomic functions like anticholinergic (including antidepressants, antihistaminics and over the counter cough and cold medications) for 2 days prior to testing; (iv) To wear comfortable and loose clothing.

2. Recording: For short term analysis of HRV, 5 minute ECG was recorded in the study participants using the (AD Instruments) hardware and Power Lab (AD Instruments) software in supine position after 15 minutes of supine rest on a bed. All recordings were done between 8-11 am in a light and noise minimized room in the department and a room temperature of $27^{\circ} \mathrm{C}$ was maintained for all the recordings. Subject was asked to close the eyes and to avoid talking, to avoid moving hands, legs or body, to avoid sleeping during test and to avoid coughing.

3. Acquisition: The ECG signal are continuously amplified, digitalized and stored in the computer for offline analysis.

4. Analysis: Spectral analysis of HRV was based on the fast fourier transformation.The time (Mean HR, RMSSD, SDNN) and spectral (LF, HF) parameters were analysed within 3 minutes time periods using Power Lab (AD Instruments) HRV software.

5. Quantification of HRV: Two methods are there in analyzing HRV: the time domain, and frequency domain. Time domain parameters: RMSSD- the root square of the mean of the squares of differences between adjacent RR intervals. SDNN-Standard deviation of the RR intervals, Frequency domain parameters: Low frequency (LF) power - obtained by integrating the power spectral density between $0.04-0.15 \mathrm{~Hz}$ indicates sympathetic and parasympathetic 
activity High frequency (HF) power obtained byintegrating the power spectral density between $0.15-0.4 \mathrm{~Hz}$ - indicates parasympathetic activity, LF/HF- obtained by dividing the LF power by the HF powerindicates sympatho-vagal balance.

\section{Statistical Analysis}

Data analysis was carried out by using Statistical Package for Social Science (SPSS Software, Version 16) package. The data following normal distribution was expressed as averaged (mean \pm standard deviation). The level of significance was fixed at $p<0.05$. Paired t test was used to determine the statistical difference in pre and post intervention. Independent $t$ test was used to determine the statistical difference between control and study group after intervention.

\section{Results}

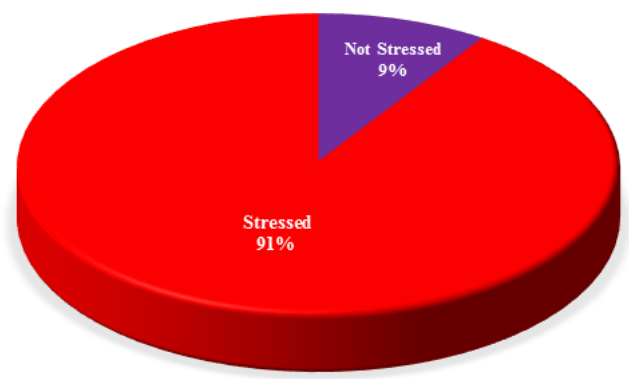

Fig 2: Stress levels in 150 first year medical students expressed in percentage

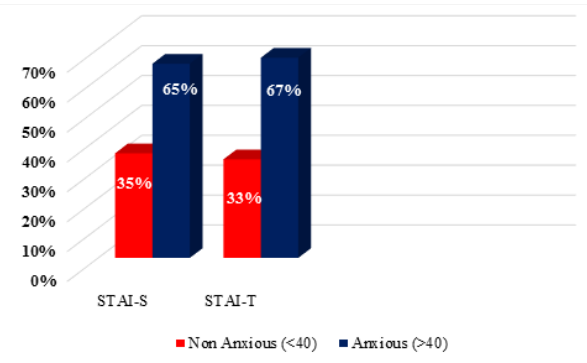

Fig 3: Anxiety state and trait levels in 150 first year medical students expressed in percentage

STAI - S - state trait anxiety inventory- state, STAI - T - state trait anxiety inventory- trait

The demographic characteristics of the subjects in study and control group were shown in Table II show no significant difference between the study and control group. In our study we found that among 150 students, $91 \%$ of the first year medical students were found to be stressed (Fig. 2), 65\% were state anxious and $67 \%$ were trait anxious (Fig 3). There was no significant difference in stress or anxiety scores and HRV in controls between baseline and after 4 weeks (Table III ). In the study group, yoga has significantly decrease in all parameters except in perceived stress scores (Table IV). At the end of 4 weeks of yoga has shown to significantly decrease in all parameters in the study group except anxiety trait when compared to the control group (Table V).

Table III: Comparison of STAI-S, STAI-T\& PSS scores \& HRV indices in control group between baseline and after 4 weeks using Paired t test

\begin{tabular}{|l|c|c|c|}
\hline Particulars & $\begin{array}{c}\text { Baseline } \\
\text { Mean } \pm \text { SD } \\
(\mathbf{n}=\mathbf{3 0})\end{array}$ & $\begin{array}{c}\text { After 4 weeks } \\
\text { Mean } \pm \mathbf{S D} \\
(\mathbf{n}=\mathbf{3 0})\end{array}$ & P value \\
\hline STAI - S & $45.87 \pm 6.296$ & $45.47 \pm 5.244$ & 0.393 \\
\hline STAI-T & $42.27 \pm 1.081$ & $42.33 \pm 2.040$ & 0.841 \\
\hline PSS & $20.63 \pm 3.64$ & $20.97 \pm 3.643$ & 0.353 \\
\hline Mean HR & $84.6 \pm 12.14$ & $84.3 \pm 10.86$ & 0.622 \\
\hline SDNN & $3.71 \pm 15.03$ & $3.69 \pm 14.82$ & 0.483 \\
\hline RMSSD & $3.12 \pm 15.26$ & $3.09 \pm 15.62$ & 0.352 \\
\hline LF in n.u. & $4.58 \pm 15.39$ & $4.41 \pm 16.02$ & 0.853 \\
\hline HF in n.u. & $2.99 \pm 11.49$ & $2.98 \pm 10.60$ & 0.254 \\
\hline LF/HF & $1.64 \pm 0.66$ & $1.40 \pm 0.093$ & 0.692 \\
\hline
\end{tabular}

All values are mean $\pm \mathrm{SD}$, STAI $-\mathrm{S}$ : state trait anxiety inventory- state, STAI $-\mathrm{T}$ :state trait anxiety inventory- trait, HR: Mean heart rate, RMSSD: square root of the mean squared difference of successive Normal to Normal RR interval, SDNN:Standard deviation of Normal to Normal RR interval, LF in n.u.: Normalized Low Frequency, HF: Normalized High Frequency. p<0.05: significant, $\mathrm{p}<0.01$ : highly significant, $\mathrm{p}<0.001$ : very highly significant. 
Table IV: Comparison of STAI-S, STAI-T \& PSS scores \& HRV indices in the study group before and after 4 weeks yoga using paired $t$ test

\begin{tabular}{|l|c|c|c|}
\hline Particulars & $\begin{array}{c}\text { Pre Yoga } \\
(\mathbf{N = 3 0})\end{array}$ & $\begin{array}{c}\text { Post Yoga } \\
(\mathbf{N}=\mathbf{3 0})\end{array}$ & P value \\
\hline STAI - S & $56 \pm 8.21$ & $40.50 \pm 6.86$ & 0.000 \\
\hline STAI-T & $56.27 \pm 8.46$ & $41.87 \pm 6.91$ & 0.000 \\
\hline PSS & $17.00 \pm 2.691$ & $15.70 \pm 4.061$ & 0.068 \\
\hline HR & $85.8 \pm 11.10$ & $76.7 \pm 11.45$ & 0.000 \\
\hline SDNN & $3.81 \pm 13.87$ & $5.43 \pm 22.78$ & 0.001 \\
\hline RMSSD & $3.84 \pm 16.79$ & $4.63 \pm 19.47$ & 0.053 \\
\hline LF in n.u. & $4.61 \pm 16.50$ & $2.80 \pm 12.49$ & 0.000 \\
\hline HF in n.u. & $3.22 \pm 11.28$ & $5.69 \pm 16.35$ & 0.000 \\
\hline LF/HF & $1.68 \pm 1.08$ & $0.55 \pm 0.34$ & 0.000 \\
\hline
\end{tabular}

All values are mean $\pm \mathrm{SD}$, STAI $-\mathrm{S}$ : state trait anxiety inventory- state, STAI $-\mathrm{T}$ :state trait anxiety inventory- trait, HR: Mean heart rate, RMSSD: square root of the mean squared difference of successive Normal to Normal RR interval, SDNN:Standard deviation of Normal to Normal RR interval, LF in n.u. : Normalized Low Frequency, HF: Normalized High Frequency. p<0.05: significant, $\mathrm{p}<0.01$ : highly significant, $\mathrm{p}<0.001$ : very highly significant.

Table V: Comparison of STAI-S, STAI-T \& PSS scores \& HRV indices between control and study groups at the end of 4 weeks

\begin{tabular}{|l|c|c|c|}
\hline & $\begin{array}{c}\text { Control Group } \\
(\mathbf{N}=\mathbf{3 0})\end{array}$ & $\begin{array}{c}\text { Study Group } \\
(\mathbf{N}=\mathbf{3 0})\end{array}$ & P Value \\
\hline STAI -S & $45.47 \pm 5.24$ & $40.20 \pm 6.86$ & 0.001 \\
\hline STAI-T & $42.33 \pm 2.04$ & $41.87 \pm 6.91$ & 0.724 \\
\hline PSS & $20.97 \pm 3.57$ & $15.70 \pm 4.06$ & 0.000 \\
\hline HR & $84.3 \pm 10.86$ & $76.7 \pm 11.45$ & 0.011 \\
\hline SDNN & $3.69 \pm 14.82$ & $5.43 \pm 22.789$ & 0.001 \\
\hline RMSSD & $3.09 \pm 15.62$ & $4.637 \pm 19.47$ & 0.001 \\
\hline $\begin{array}{l}\text { LF in } \\
\text { n.u. }\end{array}$ & $4.01 \pm 16.02$ & $2.80 \pm 12.49$ & 0.002 \\
\hline $\begin{array}{l}\text { HF in } \\
\text { n.u. }\end{array}$ & $2.98 \pm 10.60$ & $5.69 \pm 16.35$ & 0.000 \\
\hline LF/HF & $1.40 \pm 0.510$ & $0.55 \pm 0.34$ & 0.000 \\
\hline
\end{tabular}

All values are mean $\pm \mathrm{SD}$, STAI $-\mathrm{S}$ : state trait anxiety inventory- state, STAI $-\mathrm{T}$ :state trait anxiety inventory- trait, HR: Mean heart rate,RMSSD: square root of the mean squared difference of successive Normal to Normal RR interval, SDNN:Standard deviation of Normal to Normal RR interval LF in n.u.: Normalized Low Frequency, HF: Normalized High Frequency. $p<0.05$ : significant, $p<0.01$ : highly significant, $\mathrm{p}<0.001$ : very highly significant.

\section{Discussion}

Medical education is believed to be highly stressful and three important stressors are social, cultural and financial. Anxiety results in psychological problems resulting in deterioration of academic performance which further elevates anxiety and increases stress. Stress affects autonomic balance resulting in cardiac dysfunction which is assessed by HRV. Regular practice of yoga has shown to decrease mental stress and is also modulate autonomic output to achieve autonomic balance between two components (sympathetic and parasympathetic) of autonomic nervous system. ${ }^{8}$ So this study was designed to study the effect of integrated yoga on stress, anxiety and heart rate variability.

The extent of anxiety and stress is subjective, but can be quantified using State trait inventory anxiety and perceived stress scale. In our study, $91 \%$ of the first year medical students were found to be stressed (Fig. 2) which correlates with two studies from Iran and Saudi Arabia which showed stress over $60 \%$ of respondents. ${ }^{10,11}$

Our study showed a significant decline in anxiety scores from baseline after one month of yoga intervention in the study group when compared to controls (Table V). This effect of yoga was similar to other studies where yoga 
group showed significant reduction in STAI state and trait anxiety scores. ${ }^{12}$ In a non-randomized but controlled study of 50 medical students (25 yoga participants, 25 controls) over 3 months has shown significant reduction in anxiety scores following yoga practice even on the day of examinations. ${ }^{9}$ Deep relaxation technique, one of our yoga interventions in our integrated yoga schedule has been shown to decrease STAI anxiety scores in healthy volunteers by Khemka et all. ${ }^{13}$

Perceived stress score (PSS) is an index of an individual's perception to events in one's life that is perceived as stressful which helps to plan stress coping techniques like yoga. In our study, yoga intervention did not significantly decrease PSS score (Table V). This is in contrary to other studies where structured yoga has significantly decreased perceived stress, however the duration of intervention in these studies were from 12-16 weeks compared to our 4 weeks. ${ }^{14,15}$

When anxiety state and PSS scores were compared between the control and study groups after one month (Table V), we have observed a significant decrease in anxiety state levels which assesses the state of the student when the questionnaire was administered, and decrease PSS score indicating decrease in perceived stress in the previous one week with yoga intervention. This showed the usefulness of yoga in coping with stress and anxiety in spite of non-significant influence on anxiety trait of the students.

Stress and anxiety are often associated with autonomic dysfunction which adversely affects the functioning of the heart. In our study, after 4 weeks of practice of yoga, the time domain markers SDNN and RMSSD were found to be significantly increased which may be attributed to shift of autonomic balance from the sympathetic nervous system to the parasympathetic system. SDNN indicates variability in duration of diastole which in turn influences the functioning capacity of the heart. Increase in RMSSD suggests parasympathetic predominance by increased duration of cardiac cycle. ${ }^{16}$ The control group demonstrated no change in time domain parameters. In the frequency domain parameters we have been able to prove significant reduction in LF power spectrum. This may be attributed to inhibition of sympathetic area of the hypothalamus which optimizes the body's sympathetic responses to any stressful stimuli. This helps the body to restore autonomic regulatory reflex mechanisms associated with stress. It's concluded from our observations that even a one month practice of yoga reduces sympathetic tone. HF power spectrum an indicator of parasympathetic activity demonstrated a significant increase. LF/HF ratio, a marker of autonomic balance was found to be significantly reduced suggesting that there is a shift of autonomic balance towards parasympathetic predominance. Other studies shown that practice of yoga asana led to decrease in respiratory and heart rate, reduce blood pressure, lowering of cortisol levels, increase in blood flow to the intestines and vital organs, all of which points to increase in parasympathetic activity. ${ }^{17}$

In our study, we have been able to demonstrate that 4 weeks yoga intervention can significantly decrease spielberger's anxiety and trait score, and shift HRV indices to parasympathetic dominance. It may however be noted that though perceived stress was not significantly decreased with yoga, there cardiac autonomic balance was positively influenced towards parasympathetic dominance. The duration yoga intervention may not have been enough to influence perceived stress. Practice of yoga which involves lifestyle modification can be used as a non-pharmacological technique to help students to cope with stress and prevent cardiovascular complications. However it is recommend to continued practice of yoga to preserve and enhance beneficial effects obtained by short term practice of yoga.

\section{Conclusion}

One month of integrated yoga intervention in first year medical students showed significant decrease in anxiety state and improved cardio autonomic function with a shifting of sympathovagal balance towards parasympathetic dominance in heart rate variability and no significant improvement in trait anxiety.

\section{References}

1. Nirmal J, Babu CS, Harisudhan T, Ramanathan M. Evaluation of behavioural and antioxidant activity of Cytisusscoparius Link in rats exposed to chronic unpredictable mild stress. BMC Complement Altern Med. 2008;8:15.

2. Tyssen R, Vaglum P, Gronvold NT, Ekeberg O. Suicide ideation among medical students and youth physicians: a nationwide and prospective of prevalence and predictors. J Affect Disord.2001;64:69-79.

3. Tyssen R, Hem E, Vaglum P, Gronvold NT, Ekeberg $\mathrm{O}$. The process of suicidal planning among medical doctors: predictors in a longitudinal Norwegian sample. J Affect Disord. 2004;80:191-8.

4. Yusoff MS, Abdul Rahim AF, Yaacob MJ. Prevalence and Sources of Stress among UniversitiSains Malaysia Medical Students. Malays J Med Sci. 2010;17(1):30-7. 
5. Huikuri HV, Jokinen V, Syvänne $M$, Nieminen MS et al.Heart Rate Variability and Progression of Coronary Atherosclerosis. ArteriosclerThrombVascBiol 1999;19:1979-85.

6. Majercak I. The use of heart rate variability in cardiology. BratislLekListy 2002;103(10):368-77.

7. Shankarapillai R, Nair MA, George R. The effect of yoga in stress reduction for dental students performing their first periodontal surgery: A randomized controlled study. Int J Yoga. 2012;5(1):48-51.

8. Bhimani NT, Kulkarni NB, Kowale A, Salvi S. Effect of Pranayama on stress and cardiovascular autonomic function. Indian J PhysiolPharmacol. 2011;55(4):370-7.

9. Malathi A, Damodaran A. Stress due to exams in medical students--role of yoga.Indian J Physiol Pharmacol. 1999; 43(2):218-24.

10. Koochaki GM, Charkazi A, Hasanzadeh A, Saedani M, Qorbani M, Marjani A. Prevalence of stress among Iranian medical students: A questionnaire survey. East Mediterr Health J 2011;17:593-8.

11. Abdulghani HM, AlKanhal AA, Mahmoud ES, Ponnamperuma GG, Alfaris EA. Stress and its effects on medical students: A cross-sectional study at a college of medicine in Saudi Arabia. J Health PopulNutr 2011;29:516-22.

12. Javnbakht M, HejaziKenari R, Ghasemi M. Effects of yoga on depression and anxiety of women. Complement TherClinPract. 2009;15(2):102-4.

13. Khemka SS, Rao NH, Nagarathna R. Immediate effects of two relaxation techniques on healthy volunteers. Indian J PhysiolPharmacol. 2009;53(1):67-72.

14. Sharma VK, Trakroo M, Subramaniam V, Rajajeyakumar M, Bhavanani AB, Sahai A. Effect of fast and slow pranayama on perceived stress and cardiovascular parameters in young health-care students. Int J Yoga. 2013;6(2):10410.

15. Simard AA, Henry M. Impact of a short yoga intervention on medical students' health: a pilot study. Med Teach. 2009 Oct;31(10):950-2.

16. McCall T. Yoga as Medicine. New York: Bantam Dell a division of Random House Inc; 2007.

17. Woodyard C. Exploring the therapeutic effects of yoga and its ability to increase quality of life. Int J Yoga. 2011 Jul;4(2):49-54. 